\title{
Intensivmedizin: Bewährtes \& Neues als Chance für die Zukunft
}

\author{
Hugo Van Aken, Gernot Marx
}

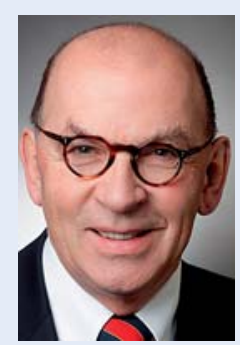

Hugo Van Aken

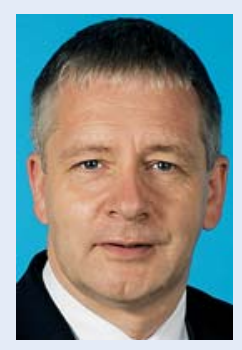

Gernot Marx

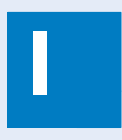

ntensivmedizin heißt sich intensiv um einen kritisch kranken Patienten kümmern - nicht mehr und nicht weniger.

Allerdings ist die Intensivmedizin ein besonders komplexer Bereich der Medizin. Um in diesem Bereich sachkundig zu arbeiten, erfordert es neben klinischer Erfahrung auch sehr viel Wissen und erfolgreiche Intensivmedizin ist immer interdisziplinäre Intensivmedizin!

Aufgrund der dynamischen und vielfältigen wissenschaftlichen Aktivitäten und neuen Forschungserkenntnisse ist man als Intensivmediziner ständig mit der Herausforderung konfrontiert, sich auf den aktuellsten Wissensstand zu bringen und zu halten, um evidenzbasiert kritisch kranke Patienten „up2date“ zu behandeln. Dies ist zweifelsohne eine schwierige Aufgabe.

Seit 2005 hat die Zeitschrift Intensivmedizin up2date sich dieser Herausforderung gestellt und exzellent gemeistert. Dies ist den vielen Autoren, dem Verlag und nicht zuletzt dem engagierten interdisziplinären Herausgeberteam zu verdanken. Nach mehr als 10 Jahren erfolgreicher Arbeit verlasse ich mit einigen Kollegen das Herausgeberteam und übergebe die wichtige Aufgabe der nächsten Generation von Intensivmedizinern. Dies schließt auch die Schriftleitung ein. Diese Aufgabe habe ich für Intensivmedizin up2date integrativ, innovativ und zielführend versucht zu erfüllen. Ich danke dem gesamten Herausgeberteam sehr herzlich für das großartige Engagement für Weiter- und Fortbildung von Intensivmedizinern in Deutschland.

Ich scheide Ende 2016 aus dem Herausgebergremium aus und übergebe die Schriftleitung zum 1.1.2017 an Herrn Professor Gernot Marx aus Aachen. Ebenso scheidet Herr Professor Tobias Welte Ende 2016 aus dem Herausgebergremium aus und übergibt seinen Platz als internistischer Herausgeber an Herrn
Professor Reimer Riessen aus Tübingen. Herr Professor Gerhard Jorch scheidet auch Ende 2016 aus dem Herausgebergremium aus und übergibt die pädiatrische Intensivmedizin an Herrn PD Sebastian Brenner aus Dresden.

Das verjüngte Herausgeberteam unter der neuen Schriftleitung von Professor Gernot Marx wird sich den Aufgaben der Zukunft stellen und mit Kontinuität und innovativer Weiterentwicklung der Intensivmedizin up2date die vielfältigen Herausforderungen, aber auch Chancen für die Entwicklung der Intensivmedizin in Weiter- und Fortbildung von Intensivmedizinerinnen erfolgreich annehmen.

Als Beispiel sei der demografische Wandel genannt: Der demografische Wandel induziert einen steigenden medizinischen Versorgungsbedarf in regional unterschiedlicher Ausprägung und Dynamik. Gleichzeitig verringert sich die Anzahl der Medizinerinnen und Mediziner [1]. Dadurch entsteht ein Versorgungsdefizit, insbesondere in dezentralen Regionen, das aus einem Missverhältnis zwischen dem Angebot und der Nachfrage an Fachkräften und medizinischen Leistungen insbesondere in der Intensivmedizin entsteht. Andererseits wird die Entwicklung der Digitalisierung durch weitreichende Vernetzung insbesondere für die Intensivmedizin auf dem Weg zur Medizin und Intensivmedizin 4.0 neue große Chancen für eine Personalisierte Medizin eröffnen. Als ein Beispiel unter vielen seien die neuen Möglichkeiten der neuen Mobilfunkgeneration 5G zu nennen. Im Vergleich zu 4G (LTE Advanced) soll 5G eine 1000-fach höhere Kapazität, 100-fach höhere Verbindungsdichte, 10-fach höhere Geschwindigkeit sowie 10-fach geringere Latenzzeiten mit Spitzendatenraten von 10-20 Gbit/s bieten [2]. Ohne Zweifel werden damit neue interdisziplinäre und fachübergreifende Strukturen und patientenorientierte Behandlungsprozesse geschaffen werden. 
Die Zukunft bleibt also spannend und das gesamte Herausgeberteam der Intensivmedizin up2date freut sich auf die Aufgabe, Ihnen wie gewohnt wichtige Entwicklungen und Inhalte für die Weiter-und Fortbildung kompetenzbasiert und sehr differenziert aufzubereiten und zu vermitteln.

Wir wünschen spannende und nützliche Lektüre Ihre
Hugo Van Aken
Huye Van Alan

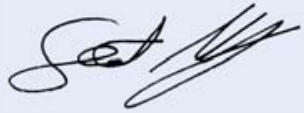

Gernot Marx

\section{Literatur}

1 Dinkelacker C, Iblher T. Ärztemangel in den Krankenhäusern: Attraktiv durch Teilzeitangebote. Dtsch Arztebl International 2011; 108: 1341 - 1342

2 5G - Initiative für Deutschland: Eine Initiative des Bundesministerium für Verkehr und digitale Infrastruktur zur Entwicklung einer Strategie zur Einführung der nächsten Mobilfunkgeneration 5G in Deutschland. https://www.bmvi.de/SharedDocs/DE/Pressemitteilungen/ 2016/154-dobrindt-5g-konferenz.html

\section{Korrespondenzadresse}

Univ.-Prof. Dr. Dr. h.c. Hugo Van Aken

ehemaliger Direktor der Klinik für Anästhesiologie, operative Intensivmedizin und Schmerztherapie Universitätsklinikum Münster

Albert-Schweitzer-Campus 1 ,

Gebäude A 1

48149 Münster

hva@uni-muenster.de

Univ.-Prof. Dr. med. Gernot Marx

Direktor der Klinik für Operative Intensivmedizin und Intermediate Care

Uniklinik RWTH Aachen

Pauwelsstr. 30

52074 Aachen

gmarx@ukaachen.de

www.operative-intensivmedizin.de 